\title{
Transient ischemic attack after Mesobuthus gibbosus envenomation
}

\author{
Yalcin Golcuk $^{1}$, Murat Ozsarac ${ }^{1}$, Adnan Bilge ${ }^{1}$, Burcu Golcuk ${ }^{2}$, Bilge Piri Cinar ${ }^{3}$, Mustafa \\ Hayran $^{1}$
}

\begin{abstract}
Mesobuthus gibbosus is a medically important venomous scorpion in western of Turkey which causes significant morbidity. Envenoming of this species scorpion results with varying degrees of local symptoms and clinically important systemic effects. This case describes an authenticated first case of transient ischemic attack in a 68 year-old-woman following envenoming by M. gibbosus in Turkey.
\end{abstract}

Keywords: Scorpion, Envenomation, Emergency department

\section{Introduction}

Scorpion envenomation and their consequences represent an important cause of emergency in certain regions of the world [1]. Mesobuthus gibbosus (also called Anatolian yellow scorpion or Mediterranean scorpion) is a member of the Buthidae family which is one of the most widely distributed and important scorpion in western of Turkey (eg Manisa, Aydın, Izmir)[2]. Scorpion envenomation can cause life - threatening complications such as respiratory, neurologic, and cardiovascular compromise [3]. We report here the first case of transient ischemic attack (TIA) following $M$. gibbosus envenoming.

\section{Case}

A 68-year-old woman with a past medical history of peptic ulcer disease was stung by a large yellow scorpion on her first finger of the right foot. She was admitted to our emergency department (ED) an hour later due to severe sharp pain, paresthesia and burning at the sting site. The scorpion was killed and brought to the ED and later identified as $M$. gibbosus. On initial examination, the patient was conscious and well oriented, and her vital signs were as follows: a blood pressure of $148 / 68 \mathrm{mmHg}$, a pulse rate of 84 beats per minute, a respiratory rate of 16 breaths per minute, a body temperature of $36.4^{\circ} \mathrm{C}$, and an oxygen saturation of $98 \%$ on room air. There was a red spot on her first digit of the right foot. Otherwise, her physical examination was unremarkable. An initial laboratory values were within the normal limits. During the ED course, the patient received intravenously $50 \mu \mathrm{g}$ fentanyl for pain relief and admitted to observation unit for any complication.
Approximately over 5 hours after envenomation, her blood pressure was progressively elevated (max level of 218/115 $\mathrm{mmHg}$ ) despite the $50 \mathrm{mg}$ captopril orally administration (prazosin was not administered, because it is not available in our hospital) and the patient developed new-onset and progressive neurologic deterioration.

In neurological examination, she had moderate dysarthria, left-sided hemiparesis with muscle power of $3 / 5$ in upper and lower extremities. The deep tendon reflexes were brisk and a left Babinski sign could be easily elicited. An urgent non-contrast computed tomography scan of the brain detected no acute hemorrhage, mass effect, or sign of acute ischemic lesion.

A single vial (dissolved in $100 \mathrm{~mL}$ of crystalloid) polyvalent scorpion antivenom (Refik Saydam Hygiene Center, Ankara, Turkey) was administered intravenously over approximately 30 minutes. Subsequent diffusion-weighted magnetic resonance imaging revealed no diffusion restriction with normal apparent diffusion coefficient values.

Following the antivenom administration, her neurologic symptoms resolved completely within 2 hours. After treatment with antivenom, bedside transthoracic echocardiography revealed mild mitral regurgitation, severe pulmonary hypertension and normal left ventricular wall motion with an ejection fraction of $60 \%$. Color Doppler of the carotid arteries examination showed a $20 \%$ right-sided internal carotid artery stenosis. She was discharged from the ED after 2 days in good clinical condition and without any neurologic sequelae. 
Discussion

Venom of $M$. gibbosus generally consists of several toxins and other compounds. The most important components of the venom are neurotoxins that primarily target voltage-gated ion channels. The NaScTx toxins bind to the resting state of $\mathrm{Na}^{+}$-channels and inhibit fast inactivation, and they induce a reduction of the peak sodium current amplitude and shift the voltage dependence of $\mathrm{Na}^{+}$-channel activation towards a more hyperpolarized membrane potential. The MegKTx toxins, recently described $\mathrm{K}^{+}$-channel specific toxins of $M$. gibbosus, bind with high affinity to the $\mathrm{K}^{+}$-channels and block the potassium permeability. Combine effect of this neurotoxins causes stabilization of voltage-gated ion channels in the open position, leading to continuous, prolonged, repetitive firing of the somatic, sympathetic, and parasympathetic neurons. As a consequence, neurotoxins result in release of excessive neurotransmitters such as epinephrine, norepinephrine, acetylcholine, glutamate, and aspartate at synapses and the neuromuscular junction [4].

Neurological complications after scorpion envenomations typically include cranial nerve dysfunctions (eg, ptosis, nystagmus, blurred vision, tongue fasciculations, slurred speech, and hypersalivation), somatic skeletal neuromuscular dysfunction (eg, restlessness, fasciculations), ischaemic or haemorrhagic strokes, and acute hypertensive encephalopathy. On the other hand, TIA is a rare complication after scorpion envenomation [2]. To the best of our knowledge, this is the first report of TIA due to M. gibbosus envenomation.

The exact pathogenesis of TIA due to $M$. gibbosus envenomation is not fully understood, but some possible mechanisms have been postulated. First of all, scorpion toxins and other venom components induce inflammation because of neutrophil infiltration occurs in different tissue, causing vasculitis, arteritis, fibrin deposition, and intravascular thrombosis [5]. Although serum catecholamine levels were not measured in our case, second possible mechanism of TIA combined with significant hypertension and tachycardia was postulated from the catecholamine-induced vasospasm that may play a role in arterial occlusion [4]. Moreover, it is also possible that, in our patient pre-existing carotid artery stenosis might act as a predisposing factor in the mechanism of $M$. gibbosus venom induced thrombotic occlusion.
In conclusion, all patients with scorpion envenomation should be closely monitored in anticipation of possible neurological complications due to direct toxic effects of the venom, vasospasm, and hypertension especially in patient with known carotid artery disease. In this case, antivenom holds a pivotal place in treating severe envenomation.

Conflict of Interest: The authors declare no potential conflicts of interest with respect to the research, authorship, and/or publication of this article.

\section{References}

1. Chippaux JP, Goyffon M. Epidemiology of scorpionism: a global appraisal. Acta tropica. 2008;107(2):71-9.

2. Koc H, Yagmur EA. Determination of Surface Activity of Mesobuthus gibbosus (Scorpiones: Buthidae) by Use of the Pitfall Traps in Dagmarmara (Turgutlu-Manisa). Ekoloji. 2007;65:60-4.

3. Bawaskar HS, Bawaskar PH. Scorpion sting: update. The Journal of the Association of Physicians of India. 2012;60:46-55.

4. Quintero-Hernandez V, Jimenez-Vargas JM, Gurrola GB, Valdivia HH, Possani LD. Scorpion venom components that affect ion-channels function. Toxicon : official journal of the International Society on Toxinology. 2013;76:328-42.

5. Petricevich VL. Scorpion venom and the inflammatory response. Mediators of inflammation. 2010;2010:903295. 